\title{
Rudeness and Rapport: Insults and Learning Gains in Peer Tutoring
}

\author{
Amy Ogan ${ }^{1}$, Samantha Finkelstein ${ }^{1}$, Erin Walker ${ }^{2}$, Ryan Carlson $^{1}$, Justine Cassell $^{1}$ \\ Human-Computer Interaction Institute, Carnegie Mellon University, Pittsburgh, PA $15213^{1}$ \\ School of Computing, CIDSE, Arizona State University, Tempe, AZ $85282^{2}$ \\ \{aeo, slfink\}@cs.cmu.edu, erin.a.walker@asu.edu, \{rcarlson, justine $\} @$ cs.cmu.edu
}

\begin{abstract}
For 20 years, researchers have envisioned artificially intelligent learning companions that evolve with their students as they grow and learn. However, while communication theory suggests that positivity decreases over time in relationships, most tutoring systems designed to build rapport with a student remain adamantly polite, and may therefore inadvertently distance the learner from the agent over time. We present an analysis of high school friends interacting in a peer tutoring environment as a step towards designing agents that sustain long-term pedagogical relationships with learners. We find that tutees and tutors use different language behaviors: tutees express more playfulness and face-threat, while tutors attend more to the task. This face-threat by the tutee is associated with increased learning gains for their tutor. Additionally, a small sample of partners who were strangers learned less than friends, and in these dyads increased face-threat was negatively correlated with learning. Our findings support the idea that learning companions should gradually move towards playful face-threat as they build relationships with their students.
\end{abstract}

Keywords: Rapport, impoliteness, virtual peers, ECA, teachable agent

\section{Introduction}

Peer tutoring, a paradigm in which one student tutors another of a similar ability, results in deep learning gains for the tutor [1]. Peer tutoring provides a social motivation for the tutor to attend more in order to effectively explain concepts [2]. In addition, the tutor engages in a series of cognitive steps that improve learning, such as constructing explanations and reflecting on errors [3]. The tutee plays an active role in this process by challenging, contradicting, and questioning the tutor's moves [3] causing the tutor to engage in increased reflection and self-explanation [1].

In the ITS community, an effort has been underway to develop virtual characters that act as a tutee, or teachable agent, in order to leverage the benefits of human peer tutoring $[4,5,6]$. However, most teachable agents focus on the cognitive elements of the interaction and, to date, none have been designed based on analyses of the social behaviors that emerge as a part of successful peer tutoring. There is therefore great opportunity to expand on the social capabilities of teachable agents in order to create

adfa, p. 1, 2011.

(C) Springer-Verlag Berlin Heidelberg 2011 
rapport in the service of increased learning. Ideally, these social teachable agents and other kinds of virtual peers - will be able to build long-term relationships with students to support them in their educational goals [as proposed in 7].

Researchers have previously designed polite intelligent tutors based on Goffman's theory of face, that is, the public self-image that people project [8]. Brown and Levinson describe positive face as the desire for one's image to be appreciated and negative face as the desire to not be impeded in one's actions [9]. Existing systems avoid threatening positive and negative face by giving praise, providing reassurance, or hedging requests $[10,11]$. However, while politeness serves a function early in a relationship, positivity is claimed to decrease as rapport increases in human-human interactions [12]. Culpeper's theory of impoliteness [13] describes the role of behaviors such as insults and challenges which are considered face-threatening; they harm the addressee's positive or negative face, and may cause offense [9]. However, impoliteness has a number of functions in conversation. It may serve to upend power imbalances [13] or even to reinforce solidarity and rapport among people with preexisting relationships $[14,15]$. Teens in particular have been shown to use "rude" language to positive social effect [16]. For that reason in this paper we evaluate the strategies and functions these language behaviors effect within particular contexts [17].

Evidence suggests that impoliteness is important in human-agent relationships as well. Our previous work demonstrated that negative remarks (such as teasing and frustration) directed to a virtual tutee in a think-aloud protocol were associated with increased learning gains on the part of the tutor [18]. There have been a few efforts to create intelligent tutoring systems that use rudeness (such as sarcasm) as a rapportbuilding mechanism [e.g, 19]. These systems were positively received by students, but were not based on analyses of human-human interaction, and learning gains were not assessed. We know that learners apply the same norms of social interaction to learning companions as to human conversational partners [20]; therefore, understanding human-human behavior is critical in the development of a system able to develop a natural social relationship with the learner over time, in the service of learning.

In this work we analyze dialogues between pairs of students participating in a peer tutoring intervention by annotating 54 conversations for language features (e.g., complaining), which we group into conversational strategies (e.g. face threat), and also code for social functionality (positivity and impoliteness). These students were friends and thus are presumed to have pre-existent relationships. We use these data to investigate two research questions: in human-human peer tutoring dialogues, can we link particular surface level language features to social conversational strategies, such as face-threat, and does this linkage differ between tutees and tutors $(R Q 1)$ ? Do these conversational strategies relate to social functions, and does this have an effect on peer tutor learning $(R Q 2)$ ? An exploratory analysis of 6 dyads of strangers (presumed not to have prior relationships) allows us to address a third research question that may provide insight into the design of relationship-building systems that evolve over time: How do the relationship-affecting conversational strategies of strangers relate to learning, and differ from friends (RQ3)? Our results yield specific design guidelines for implementing relationship-building behaviors in an interactive tutoring system specifically, a teachable agent, grounded in our findings from human-human tutoring. 


\section{Study}

To assess the social behaviors of real students in a peer tutoring context, we reexamined data collected for a previous study to evaluate the impact of an intervention that monitored students' collaboration and could provide adaptive support [21]. A peer tutor and tutee interacted over chat while the tutee worked on algebra problems. Participants were $1308^{\text {th }}-10^{\text {th }}$ grade students (49 male) with diverse racial backgrounds from one American high school who had previously received classroom instruction on relevant domain material. Participants were asked to sign up for the study with a friend. Those who were interested but had no partner were matched with another unmatched participant. 54 dyads were friends and 6 dyads were strangers. Participants took a 20-minute pre-test on relevant math concepts, and then spent 20 minutes working alone with the computer to prepare for tutoring. One student in each dyad was randomly assigned the role of tutor, while the other was given the role of tutee. They spent the next 60 minutes engaging in tutoring. Finally, students were given a domain post-test isomorphic to the pretest, and compensated.

\section{Data annotation}

We analyzed the tutoring dialogues using a scheme we developed to capture three levels of relationship-building and signaling: specific language behaviors, the conversational strategies they contribute to, and their associated social functions. The distinction between these three levels was drawn from work in pragmatics [8] that allows us to interpret the different social functions of groups of language behaviors used in context (such as insults used to indicate solidarity and therefore build rapport, or politeness used to indicate distance and therefore push away) [17]. Much of our analysis focuses on the friend dyads: 5,408 utterances from 108 participants over 54 sessions. 2,333 of these utterances were produced by the tutee and 3,075 by the tutor.

Thirteen surface-level language behaviors, shown in Table 1, were coded by two independent raters, based on research on impoliteness [13], positivity in tutorial dialogues [22], and computer-mediated communication [23]. Each utterance could receive more than one code. Counts of features were normalized by the total number of utterances spoken by that participant. Based on the Principal Components Analysis presented in section 4.1 below, codes were grouped into factors representing conversational strategies. Each utterance was also annotated for two types of social functions, motivated by the literature on rapport-building and -maintaining. This entailed examining the interlocutor's response to a given act; the same utterance may serve a different social function depending on its reception. The positivity code was expanded beyond politeness to encompass other indicators of positivity such as those used by Boyer [22] including empathy, praise, and reassurance, in addition to cooperative talk. $\left(\mathrm{M}_{\text {tutor }}=14 \% ; \mathrm{M}_{\text {tutee }}=17 \%\right.$; Cohen's $\left.\mathrm{K}=.79\right)$. The impoliteness code expresses negativity, combining both cooperative rudeness such as teasing and banter (e.g. "I hate youuuu :D"), and uncooperative rudeness which seems to intend to cause offense (e.g., "your horrible at this.") [15] ( $\mathrm{M}_{\text {tutor }}=8 \% ; \mathrm{M}_{\text {tutee }}=12 \%$; Cohen's $\left.\mathrm{K}=.72\right)$. 


\section{$4 \quad$ Results}

\subsection{Surface-level language features and role differences $(R Q 1)$}

Table 1. Annotation scheme divided into factors, with mean normalized behaviors for tutors and tutees, and Cohen's kappa between raters. Significantly higher values (comparing tutor to tutee) marked with $*(\mathrm{p}<.05), * *(\mathrm{p}<.01), * * *(\mathrm{p}<.001)$.

\begin{tabular}{|c|c|c|c|c|c|c|}
\hline \multicolumn{2}{|l|}{ Factor 1: Playfulness } & \multirow{2}{*}{$\begin{array}{l}\mathbf{K} \\
1\end{array}$} & \multirow{2}{*}{$\begin{array}{c}\text { Tutor M } \\
4.3 \%\end{array}$} & \multirow{2}{*}{$\begin{array}{c}\text { Tutor SD } \\
.07\end{array}$} & \multirow{2}{*}{$\begin{array}{c}\text { Tutee } \mathbf{M} \\
6.2 \%\end{array}$} & \multirow{2}{*}{$\begin{array}{c}\text { Tutee SD } \\
.08\end{array}$} \\
\hline Laughter (L) & “hahaha," “lol!” & & & & & \\
\hline Extra letters (EL) & "tutor help meeeee" & .94 & $4.8 \%$ & .07 & $7.2 \%$ & .10 \\
\hline Emoticons (E) & "h8u <3," "did it! :-D” & 1 & $2.3 \%$ & .04 & $3.4 \%$ & .05 \\
\hline Inc. complain (I) & "this thing is such butt" & .78 & $4.8 \%$ & .05 & $8.4 \% * *$ & .08 \\
\hline Off-task (O) & "I am so hungryyyyy!” & .71 & $7.3 \%$ & .08 & $9.9 \%$ & .11 \\
\hline \multicolumn{2}{|l|}{ Factor 2: Face-threat } & & & & & \\
\hline Direct insult (DI) & "you're being so weird." & 1 & $1.3 \%$ & .02 & $2.3 \%$ & .03 \\
\hline Condescension $(\mathrm{C})$ & “... obviously add now. Duh.” & 1 & $0.9 \%$ & .02 & $2.9 \% * * *$ & .04 \\
\hline Challenge (Ch) & $\begin{array}{l}\text { tutor: okay bro now subtract" } \\
\text { tutee: "noo, not right, I add." }\end{array}$ & .91 & $3.0 \%$ & .03 & $5.6 \% * *$ & .06 \\
\hline Excl. complain (EC) & "you're making no sense dude" & 8 & $1.2 \%$ & .02 & $3.4 \% * * *$ & .04 \\
\hline \multicolumn{2}{|l|}{ Factor 3: Attention } & & & & & \\
\hline Enforcer (Ef) & "pay attention - now divide." & .85 & $1.7 \%^{* * *}$ & .02 & $.39 \%$ & .01 \\
\hline Vocatives (V) & "homie, that's not right." & .9 & $3.0 \%$ & .04 & $5.0 \%$ & .06 \\
\hline \multicolumn{2}{|l|}{ Factor 4: Emphasis } & & & & & \\
\hline Punctuation (P) & "I don't even see an rt?!?!?!" & .89 & $6.5 \%$ & .09 & $9.9 \%$ & .12 \\
\hline Capitalization (Ca) & "I SAID you ADD THE BD!!" & 1 & $3.1 \%$ & .06 & $5.9 \%$ & .11 \\
\hline
\end{tabular}

With the goal of understanding how surface-level language features contributed to social conversational strategies in peer tutoring dialogue, and what strategies were most frequent, we performed a Principal Components Analysis (PCA) with Varimax rotation. This allowed us to move from a focus on individual behaviors to understanding how particular types of behaviors are used in meaningful ways in this population. The annotated language features collapsed into four factors, which explained $76 \%$ of the total variance. Table 1 shows the mapping between the language features and factors. Based on the pragmatics theory cited, we interpret the four factors as follows:

Playfulness to lighten the mood or mitigate negativity: Laughter, extra letters, emoticons, off-task behavior, inclusive complaining (about a third person).

Face-threat remarks directed toward the partner: Direct insults, condescension/brags, challenges, exclusive complaining (about the other person).

Attention-getting to draw the partner back on task: Message enforcers, pet names.

Emphasis to add emotive features: Excessive punctuation, capitalization.

The PCA allowed us to compute a regression value for each of these four factors for each participant, which represented their utterances in terms of these values (e.g., the 
total 'face threat' value of the conversation). We then investigated how tutee and tutor utterances differed along these factors by running a MANOVA with role as the independent variable, and four dependent variables: playfulness, face threat, attention, and emphasis. We found that tutees used more playful $(\mathrm{F}(1,107)=8.33, \mathrm{p}<.01)$ and face-threatening strategies $(\mathrm{F}(1,107)=16.62, \mathrm{p}<.001)$, while tutors used more attention-getting strategies $(\mathrm{F}(1,107)=9.72, \mathrm{p}<.01)$. Emphasis use was equivalent $(\mathrm{p}>.1)$.

These results indicate that tutees are responsible for introducing playful and facethreatening strategies in the conversation, while tutors instead bring attention back to the task. The following is a representative example from the corpus:

[] Tutee: I need help tuter

[ ] Tutor: What do you do next?

[E,DI,Ch,Ca] Tutee: it told me aks why you got it wrong. ANSWER: your stupid XD

[Ef,V,P,Ca] Tutor: dude! STOP! Add VT to both sids!

Typically, tutees' requests for help involve excessive punctuation or extra letters, both shown to contribute to "playfulness in language" in texting [23]. Tutors respond to these requests with on-task utterances (e.g. "now you need to add gh to both sides.") If tutees reply with face-threat, tutors use vocatives and message enforcers to bring the conversation back on task. We explore this interplay further in section 4.3.

\subsection{Conversational strategies and social functions (RQ1)}

With the results of the PCA, we examined the social conversational strategies effected by off-task social language such as complaining and exclamations. We next analyze the social functions of expressions of positivity and impoliteness in particular, and the relationship between conversational strategies and social functions. We investigated tutor and tutee differences using a MANOVA with role as the independent variable, and positivity and impoliteness as the dependent variables. Given the PCA results, it is not surprising to find that tutees were significantly more impolite $(F(1,107)=7.74$, $\mathrm{p}<.01)$ than tutors, and marginally less positive $(\mathrm{F}(1,107)=3.60, \mathrm{p}=.06)$.

We thus analyzed the connection between conversational strategies and social functions separately for tutors and tutees using bivariate correlations. While the tutees primarily expressed positivity with playfulness $(\mathrm{r}=.276, \mathrm{p}<.05)$, tutors expressed positivity with emphasis $(\mathrm{r}=.359, \mathrm{p}<.01)$ in addition to playfulness $(\mathrm{r}=.436, \mathrm{p}=.001)$. That is, tutees primarily achieved conversational positivity through playful non-standard writing, complaining about the task they were doing, or interjecting off-task comments into the dialogue. Tutors used these techniques, but additionally expressed positivity through emphasizing their utterances with excess punctuation and using the caps lock, such as to praise their partner (e.g. "YAY you DID IT!!!").

Differences were also apparent in impoliteness, which tutees primarily expressed through face-threatening features $(\mathrm{r}=.5, \mathrm{p}<.001)$ and attention-getting features $(\mathrm{r}=.306, \mathrm{p}<.05)$. In contrast, tutors used only attention-getting features such as message enforcers to indicate impoliteness $(\mathrm{r}=.517, \mathrm{p}<.001)$. These correlations are supported by qualitative analyses of the data, such as the following example where the tutor continues to keep the conversation on task despite the tutees' face threat. 
[DI, C, EC] Tutee: your horrible at this

[Ef] Tutor: thanks... i try. Just restart the problem.

[EC] Tutee: can you actually say something that i can fully understand

[ ] Tutor: add vt then you have to solve for t so subtract bh from both sides

Despite these apparent differences, a correlation was found in the language use of tutor-tutee pairs, ranging from a weak correlation for attention-getting $(\mathrm{r}=.244, \mathrm{p}<.05)$ to very strong correlations, e.g., playfulness $(\mathrm{r}=.840, \mathrm{p}<.001)$. This result indicates synchrony or coordination in the dyads, a marker of the kind of rapport that characterizes long-term relationships [12]. So while partners identify their roles (tutor or tutee) through conversational strategies, they also index their rapport by not straying too far from the partner's language patterns.

\subsection{Learning gains, language features, and social functions ( $R Q 2)$}

Our second research question investigates how language use relates to learning outcomes. To address the design of teachable agents, we examined the relation between tutees' behaviors and their partner's learning gains. A stepwise regression looking at the four conversational strategies, the social functions, and the interactions among these features $\left.\left(\mathrm{r}^{2}=.07, \mathrm{~F}(1,107)=1.824, \mathrm{p}=.1\right)\right)$ found that face threat is a positive predictor of learning gains $(\beta=.375, t=2.22 \mathrm{p}=.03)$, while the interaction term of face threat $\mathrm{x}$ positivity is a negative predictor of learning gains $(\beta=-.320, t=-1.86, p=.06)$. This means that as face threat increases, tutors learn more, and the learning benefits of face threat can be enhanced by appropriate use of positivity. In essence, face threatening conversational strategies with socially positive functions enhance the learning interaction. On the other hand, high positivity with low face threat from the tutee is actually associated with lower levels of learning. That is, positive social interaction that does not contain the kind of face-threatening behavior that characterizes rapport in this age group may either signal less rapport, or actually reduce the connection between the dyad, and therefore reduce learning gains. In addition, a lack of facethreatening interactions may indicate a lack of the challenging tutor moves by the tutee, that increase the cognitive benefits of the interaction.

In order to explore how these functions were associated with learning, we quantified how tutors reacted to the use of positivity and face threat by the tutee. We used transition matrices to evaluate the conditional probability of a feature occurring in one turn based on the presence of another in the prior turn (collapsing consecutive utterances by the same speaker to form turns). Thus, we calculate the probability that the tutor will use feature $B$ given that the tutee used feature $A$ in the previous turn. By examining these transition matrices (see Table 2 for values), we can identify common response patterns in the dyads. We found that when the tutee exhibits positivity, the tutor is no more likely to respond with positivity (42\%) than with a response that contains no coded social features $(46 \%)$. Generally, these instances with no codes are task-related, non-emotive statements such as "ok add five". When a tutee exhibits face-threat, on the other hand, the tutor is more likely (57\%) to respond with no social features (indicating that the tutor is likely using task features). Thus, while tutors 
demonstrate no particular pattern of response to positivity, they are likely to respond to negativity with strategies to keep the conversation on-task. Negative behaviors such as face-threats on the part of the tutor therefore are more likely to elicit effective tutoring behaviors than are positive behaviors such as praise.

Reversing the direction of the conditional probability demonstrated that while tutee behavior with respect to positivity is similar to observed tutor behavior, tutee behavior when the tutor exhibits face threat is very different. Whereas a tutor is not likely to engage her tutee, the tutee is just as likely to fire back with impoliteness $(36 \%)$ as she is to refrain $(33 \%)$. The imbalance of power between the two roles within the context of an existent friendship may lead the tutee to try to regain the upper hand through face-threat, while the tutor tries to regain authority through task behavior.

Table 2. Selected entries from transition matrix, for friends. Left-most column shows initiator and language feature exhibited. Transition percentages indicate number of times feature was seen in partner's response, divided by the total number of partner responses to feature. Because features can co-occur in an utterance, response percentages may not always sum to 1 .

\section{Partner response}

\begin{tabular}{ll|lccc}
\multicolumn{2}{l}{} & None (\%) & Positivity (\%) & Impoliteness (\%) & Off-topic (\%) \\
\hline \multirow{2}{*}{ Tutee } & Positivity & 46 & 42 & 8 & 24 \\
& Face threat & 57 & 19 & 23 & 15 \\
\hline Tutor & Positivity & 38 & 42 & 11 & 23 \\
& Face threat & 33 & 16 & 36 & 19
\end{tabular}

\subsection{Relationships: Friends and strangers $(R Q 3)$}

In addition to the fifty-four dyads analyzed above, six dyads participated in the study who either did not sign up with a friend, or whose schedule changed requiring them to be partnered with another unmatched participant. Given literature that suggests that the demonstration of rapport differs between friends and strangers (those who are building rather than maintaining rapport) [13], these dyads provide a contrast to the data from partners who were already friends.

An ANOVA with role and friend as independent variables and partner learning gains as the dependent variable shows that friends had significantly greater learning gains than strangers $\left(\mathrm{F}(1,120)=4.71, \mathrm{p}=.03 ; \mathrm{M}_{\text {stranger }}=-.17, \mathrm{SD}_{\text {stranger }}=.35, \mathrm{M}_{\text {friend }}=.02\right.$, $\mathrm{SD}_{\text {friend }}=.28$ ), while role was not significant in this analysis ( $\mathrm{p}>.1$ ). Given that strangers tended to learn less from the intervention, we investigated whether the factors related to their learning were equivalent to those of friends. A stepwise regression showed that face threat is a strong negative predictor of learning gains for strangers (overall model: $\mathrm{r}^{2}=.44, \mathrm{~F}(1,11)=8.516, \mathrm{p}=.015$; effects of face threat: $\beta=-.678, \mathrm{t}=-$ $2.92, \mathrm{p}=.015)$. In other words, in direct contrast to friends, greater amounts of facethreatening behaviors by non-friend tutees actually do threaten the relationship, and hence are associated with lower learning gains for the tutor.

The behavior transition matrices demonstrate that, for strangers, in every possible transition, a response containing no coded features was more likely than any other behavior. That is, strangers tend to produce task-related, non-emotive statements in 
response to all other behaviors. Furthermore, when the stranger tutee exhibits face threat towards the tutor, only $14 \%$ of the time will the tutor reply with impoliteness, with other transitions producing similar results. It is also notable that there are only three instances of face threat from the stranger tutors, and in each case the tutee responds with no coded features. Strangers are also much more hesitant to respond to positivity. Compared to friends, we see strangers responding with a much more restricted set of behaviors, suggesting a discomfort with confrontation not seen in friend dyads [24]. When face threat does happen, it is not beneficial for learning.

\section{$5 \quad$ Discussion and conclusions}

Though most intelligent tutoring systems that attempt to build rapport with the learner do so through politeness, actual peer tutors employ a great deal of impolite and facethreatening behavior. In this paper, we have analyzed chat data from a computersupported peer tutoring intervention to investigate how peer tutors and tutees use surface-level language features to contribute to a set of particularly teen-like communicative strategies. These strategies interact with positive and negative social relationship functions in ways which correlate with learning gains. Importantly, the preexisting social relationships between the partners also matter, as this chain of effects differs in interesting ways between friends and strangers.

Through a factor analysis that investigated groupings of thirteen language features, we determined ways in which peer tutors and tutees use these various features to accomplish playfulness, face-threat, attention-getting, and emphasis communicative strategies. Understanding how students use the same features to achieve different positive and negative communicative strategies within a dialogue will allow us to develop teachable agents who are able to index the language features of a community to respond appropriately to their partner both socially and cognitively.

An investigation of how tutors and tutees differentially use these communicative strategies showed that tutees tend to be responsible for the bulk of positive and negative social input in a dialogue, while tutors keep the interaction on track by directing the tutee's attention. Yet, tutors and tutees do act in synchrony, with dyads displaying correlated levels of each of the four communicative strategies, and their consequent social functions such as positivity and impoliteness. The synchrony between the partners is an index of their friendship, while the asynchrony in the use of social language - and negativity in particular - may be demonstrating an attempt by the tutee to redress the power differential of the two roles, and by the tutor to maintain the higher status of instructor. This conflict, however, keeps within the frame of friendship as demonstrated by the lack of negative response by the tutor to the tutee's insults.

It is undoubtedly the fact that the friendship supports - or even thrives on - so much apparent negativity that leads to our result that increased face threat on the tutee's part leads to increased learning on the tutor's part. Tutees are keeping the tutors on their intellectual toes by challenging their help, demanding explanations, and questioning their methods [3]. What we show in this work is that these playground strategies of playful insults, criticisms, and condescension [16] can serve the same goal as 
the challenges and contradictions that mark good peer tutoring [3]. It is likely, however, that impoliteness has its limits; excessive criticism or insult may fail at both social and cognitive goals. Accordingly, we find that positivity also plays a critical role in these tutoring interactions, as it enhanced the learning benefits of face threatening acts, while it was not associated with learning on its own. The interactions between these factors are complex, and leave ample room for future work.

Though preliminary analyses indicate that even strangers will use some facethreatening behaviors during tutorial dialogues, among these dyads, the presence of such behaviors leads to reduced learning gains for both the tutor and the tutee. We cannot and should not assume that a teachable agent and its tutor begin as friends. Thus, in the design of such agents, we will want to investigate the effectiveness of a model that begins with very few face-threatening behaviors. Neither should we abandon hope, however, that the agent and his tutor will embark on a relationship over time. We therefore propose that over multiple sessions, the agent begin to drive the learning by becoming increasingly face-threatening through challenges and even insults, while maintaining a synchrony with the tutor's usage of face-threat in return.

As friendship between partners was not randomly controlled in this data, future work should investigate tutor-tutee rapport between friends and strangers in a more controlled setting. And as children perform many more social moves than we coded, future directions should examine additional behaviors, such as a breakdown of politeness moves typically referenced in intelligent tutoring systems [10]. It is also important to note that our data is rooted within a particular context; specifically, that of teenage American students interacting through a textual interface. While our results may not generalize beyond this context, it is fortunately one ripe for research in educational technology. In any case, if tutoring systems and virtual peers are to play a role as long-term learning companions, they must have the ability to evoke, signal and maintain relationships in ways appropriate to the age group they are built for, and that they must be capable of changing those relational strategies over time.

Acknowledgements. This research was supported by NSF Awards DRL-0910176 and IIS-0968485 and the IES, U.S. Dept. of Ed., through R305A090519 to Carnegie Mellon University. It was also supported by the Pittsburgh Science of Learning Center, funded by NSF Award SBE-0836012. The opinions expressed are those of the authors and do not represent views of the funders.

\section{References}

1. Sharpley, A., Irvine, J., Sharpley, C. "An examination of the effectiveness of a cross-age tutoring program in mathematics for elementary school children." American Educational Research Journal, 20(1), 1983: 101-111.

2. Rohrbeck, C. A. Ginsburg-Block, Marika, D., Fantuzzo, J.W., Miller, T. R. "Peer-assisted learning interventions with elementary school students: a meta-analytic review." Journal of Educational Society, 95(2)., 2003: 240-257.

3. Webb, Noreen. "Peer interaction and learning in small groups." International Journal of Educational Research, 13(1), 1989: 21-39. 
4. Brophy, S., Biswas, G., Katzberger, T., Bransford, J., Schwartz, D. "Teachable agents: combining insights from learning theory and computer science." Artificial Intelligence in Education, 50. 1999: 21-28.

5. Matsuda, B., Yarzebinski, E., Keiser, V., Raizada, R., Stylianides, G., Cohen, W. W. "Learning by Teaching SimStudent - an initial classroom baseline study with cognitive tutor." Proceedings of Artificial Intelligence in Education. Springer, 2011. 213-221.

6. Gulz, A., Silvervarg, A., Sjoden, B. "Design for off-task interaction - Rethinking pedagogy in technology enhanced learning." Proceedings of the 10th IEEE International Conference on Advanced Learning Technologies. 2010.

7. Chan, T., Baskin, A. "Studying with the prince: the computer as a learning companion." ITS '88 Conference. Montreal, Canada, 1988. 194-200.

8. Goffman, Erving. The presentation of self in everyday life. NY: Doubleday, 1959.

9. Brown, P, and S Levinson. "Universals in Language Usage: Politeness phenomena." In Questions and politeness: Strategies in social interaction, by E. N. Goody (ed). London: Cambridge: University Press, 1978.

10. McLaren, B., DeLeeuw, K., Mayer, R. "Polite web-based intelligent tutors: Can they improve learning in classrooms?" Computers and Education, 56(3), 2011: 574-584.

11. Johnson, L., Paola, R. Politeness in tutoring dialogs: "Run the factory, that's what I'd do." Intelligent Tutoring Systems, 206-243. 2004.

12. Tickle-Degnen, Linda, and Robert Rosenthal. "The Nature of Rapport and its Nonverbal Correlates." Psychological Inquiry, 1990: 285-293.

13. Culpeper, J. Towards an anatomy of impoliteness. Journal of Pragmatics, 25(3). 1996. 349-367.

14. Straehle, C. A. "'Samuel?" "Yes dear?" Teasing and conversational rapport." In Framing in Discourse, by D. Tannen. New York: Open University Press, 1993.

15. Keinpointer, M. "Varieties of rudeness: types and functions of impolite utterances." Functions of Language, 1997: 251-287.

16. Ardington, A. Playfully negotiated activity in girls' talk, Journal of Pragmatics, 38(1) (2006), 73-95.

17. Mills, S. "Gender and politeness" Journal of Politeness Research 1(2): 263-280 (2005).

18. Ogan, A., Finkelstein, S., Mayfield, E., D’Adamo, C., Matsuda, N., Cassell, J. (2012). "Oh dear Stacy!" Social Interaction, Elaboration, and Learning with Teachable Agents. To appear in Proceedings of CHI 2012.

19. Graesser, A., McNamara, D. "Self-Regulated Learning in Learning Environments with Pedagogical Agents that Interact in Natural Language." The Measurement of Learners' Self-Regulated Cognitive and Metacognitive Processes While Using Computer-Based Learning Environments, 2010: 234-244.

20. Cassell, J (2004) "Towards a Model of Technology and Literacy Development: Story Listening Systems” Journal of Applied Developmental Psychology 25 (1): 75-105.

21. Walker, E., Rummel, N., \& Koedinger, K. R. (2011). Adaptive support for CSCL: Is it feedback relevance or increased accountability that matters? In Proceedings of the 10th International Conference on Computer-Supported Collaborative Learning (pp. 334-342).

22. Boyer, K., Phillips, R., Wallis, M., Vouk, M., Lester, J. "Balancing cognitive and motivational scaffolding in tutorial dialogue." Intelligent Tutoring Systems. 2008. 239-249.

23. Herring, S., Zelenkauskaite, A. "Symbolic Capital in a Virtual Heterosexual Market." Written Communication, 26, 2009: 5-31.

24. Cassell, J., Gill, A. \& Tepper, P. (2007) Coordination in Conversation and Rapport. Proceedings of the Workshop on Embodied Natural Language, Association for Computational Linguistics. 